Acta Crystallographica Section D

\section{Biological \\ Crystallography}

ISSN 0907-4449

Przemyslaw Nogly, ${ }^{\text {a Pedro }}$ M. Matias, ${ }^{a}$ Matteo de Rosa, Rute Castro, ${ }^{a}$ Helena Santos, ${ }^{\text {a }}$ Ana Rute Neves ${ }^{\mathrm{a}}$ and Margarida Archer $^{\mathrm{a} *}$

anstituto de Tecnologia Química e Biológica, Universidade Nova de Lisboa (ITQB-UNL), 2780-157 Oeiras, Portugal, and ${ }^{\mathbf{b}}$ Instituto Gulbenkian de Ciência (IGC), 2780-156 Oeiras, Portugal

Correspondence e-mail: archer@itqb.unl.pt

\title{
High-resolution structure of an atypical a-phosphoglucomutase related to eukaryotic phosphomannomutases
}

The first structure of a bacterial $\alpha$-phosphoglucomutase with an overall fold similar to eukaryotic phosphomannomutases is reported. Unlike most $\alpha$-phosphoglucomutases within the $\alpha$-D-phosphohexomutase superfamily, it belongs to subclass IIb of the haloacid dehalogenase superfamily (HADSF). It catalyzes the reversible conversion of $\alpha$-glucose 1 -phosphate to glucose 6-phosphate. The crystal structure of $\alpha$-phosphoglucomutase from Lactococcus lactis (APGM) was determined at $1.5 \AA$ resolution and contains a sulfate and a glycerol bound at the enzyme active site that partially mimic the substrate. A dimeric form of APGM is present in the crystal and in solution, an arrangement that may be functionally relevant. The catalytic mechanism of APGM and its strict specificity towards $\alpha$-glucose 1-phosphate are discussed.

\section{Introduction}

$\alpha$-Phosphoglucomutase ( $\alpha$-PGM; EC 5.4.2.2) is present in all organisms from bacteria to animals and plants (Whitehouse et al., 1998). This enzyme catalyzes the interconversion of $\alpha$-glucose 1-phosphate to glucose 6-phosphate and plays distinct roles in different organisms. In animals and plants, $\alpha$-PGM is mainly involved in the synthesis and utilization of storage carbohydrates such as glycogen and starch (VillarPalasi \& Larner, 1970; Fettke et al., 2009). In bacteria, $\alpha$-PGM is involved in sugar utilization and also in the synthesis of UDP-glucose, a sugar donor for the production of glucosecontaining polysaccharides. In the last decade, a large number of studies have associated the virulence of several pathogenic bacteria with the presence of $\alpha$-PGM (Plant et al., 2006; Paterson et al., 2009).

Lactococcus lactis is a lactic acid bacterium that is widely used in starter cultures for the manufacture of fermented dairy products such as cheese and buttermilk. Its enormous economic value has rendered this typical homofermentative bacterium as one of the most extensively studied members of the lactic acid bacteria. L. lactis possesses two distinct PGMs with specificity for $\alpha$ - or $\beta$-anomers of phosphoglucose. $\beta$-PGM, a member of haloacid dehalogenase superfamily (HADSF) class I, catalyzes the reversible conversion of $\beta$-glucose 1-phosphate to glucose 6-phosphate and is involved in the degradation pathways of maltose and trehalose (Andersson et al., 2001; Levander et al., 2001; Lahiri et al., 2002). $\alpha$-PGM of L. lactis (APGM) catalyzes the interconversion of $\alpha$-glucose 1-phosphate to glucose 6-phosphate and is essential in the utilization of galactose via the Leloir pathway (Grossiord et al., 1998; Neves et al., 2006). APGM has also been implicated in the synthesis of precursors of cell-wall polysaccharides and exopolysaccharides (Delcour et al., 1999;
Received 20 May 2013

Accepted 19 June 2013

PDB Reference: $\alpha$-phosphoglucomutase, 4 bnd 
Kleerebezem et al., 1999). Therefore, APGM plays an important role in the processes of galactose degradation and the formation of precursors for biosynthetic pathways during growth on glucose or other carbohydrates.

Most of the bacterial and eukaryotic $\alpha$-PGMs that utilize $\alpha$-glucose 1-phosphate belong to the $\alpha$-D-phosphohexomutase superfamily (IPR005841). These are enzymes consisting of four domains that are usually observed as monomers with a molecular mass larger than $45 \mathrm{kDa}$ (Shackelford et al., 2004). A conserved serine is utilized as a catalytic residue that is phosphorylated during the course of reaction. APGM shares no sequence or structure similarity with the common $\alpha$-PGMs of the $\alpha$-D-phosphohexomutase superfamily.

In contrast to the known bacterial $\alpha$-PGMs, the lactococcal APGM enzyme belongs to the HADSF (Neves et al., 2006). The HADSF is composed of more than 3000 proteins, which are mainly involved in phosphoryl-transfer reactions (Allen \& Dunaway-Mariano, 2004). Members of the HADSF are typically composed of two domains: the core and cap domains. The core domain contains most of the conserved catalytic residues, while the cap domain acts as a lid over the core domain and differentiates according to specific substrates and catalytic reactions (Lahiri et al., 2004; Allen \& Dunaway-Mariano, 2009). An example of a strictly conserved amino-acid residue is the aspartate that acts as a nucleophile and mediates the transfer of the phosphoryl group (Asp8 in APGM). Members of the HADSF also share a catalytic requirement for a metal cofactor, usually $\mathrm{Mg}^{2+}$. Phylogenetic studies of HADSF proteins have suggested a common origin for APGM and the eukaryotic phosphomannomutases (PMMs; IPR005002; Neves et al., 2006). However, APGM shows strict specificity for $\alpha$-glucose 1-phosphate, whereas the eukaryotic phosphomannomutases generally use both $\alpha$-mannose 1-phosphate and $\alpha$-glucose 1-phosphate as substrates (Pirard et al., 1999; Neves et al., 2006). Notably, the sequence identity between $\alpha$-phosphoglucomutase and $\beta$-phosphoglucomutase from L. lactis is only $10 \%$ (they belong to distinct clades of the phylogenetic tree; Neves et al., 2006) and results in significant structural differences, including different topologies and positions of the cap domain in the sequence. These observations suggest that $L$. lactis APGM is mechanistically closer to the PMMs than to the $\beta$-PGMs.

In this work, we report the first crystal structure of APGM. It was determined by the single-wavelength anomalous dispersion (SAD) method based on a Pt derivative and was refined to $1.5 \AA$ resolution. The structure is compared with other members of the HADSF and specific features of the enzyme with impact on substrate specificity and catalytic mechanism are discussed.

\section{Materials and methods}

\subsection{Crystallization}

The expression, purification and crystallization of APGM were performed as described previously (Nogly et al., 2012). Briefly, the pgmH gene coding for APGM in L. lactis
(Q00G41) was cloned into pNZ8048 vector with a $\mathrm{His}_{6}$ tag at the $\mathrm{N}$-terminus followed by an enterokinase cleavage site. The APGM construct of $29.9 \mathrm{kDa}$ was homologously expressed in L. lactis and purified by metal-affinity (Ni-NTA) chromatography. Crystals appeared in $2 \mathrm{M}$ ammonium sulfate, $0.1 \mathrm{M}$ HEPES $\mathrm{pH} 7.5,2 \%$ PEG 400 using the vapour-diffusion method at room temperature. Subsequently, streak-seeding from the initial multiple crystals was used to grow suitable crystals for X-ray diffraction. Crystals were cryoprotected with the crystallization solution supplemented with $20 \%$ glycerol and flash-cooled in liquid nitrogen.

Native crystals were soaked in crystallization solutions supplemented with heavy-atom compounds at room temperature. The soaking times were $20 \mathrm{~h}$ for potassium tetrachloroplatinate(II), potassium tetranitroplatinate(II), trimethyllead acetate, thiomersal and potassium gold(III) chloride, and one month for mercury(II) acetate and cisplatin. The final concentrations of these compounds ranged from 2.5 to $15 \mathrm{~m} M$.

\subsection{Gel filtration}

Size-exclusion chromatography was carried out to estimate the molecular mass of APGM in solution using a Superdex 200 16/60 gel-filtration column equilibrated with $50 \mathrm{~m} M$ HEPES $\mathrm{pH} 7.4,150 \mathrm{~m} M \mathrm{NaCl}$. The calibration curve was prepared using cytochrome $c$, carbonic anhydrase, albumin (BSA), alcohol dehydrogenase and $\beta$-amylase as standards.

\subsection{Data collection and processing}

X-ray diffraction data for APGM native crystals were collected on the ID14-2 beamline of the European Synchrotron Radiation Facility (ESRF), Grenoble, France at $100 \mathrm{~K}$. The best crystal diffracted to $1.5 \AA$ resolution and the data were integrated using MOSFLM (Battye et al., 2011) and scaled with SCALA (Evans, 2011; Nogly et al., 2012). A data set from the crystal derivatized with $\mathrm{K}_{2} \mathrm{PtCl}_{4}$ was collected to $2.4 \AA$ resolution on the I04 beamline of the Diamond Light Source (DLS), Didcot, England at $100 \mathrm{~K}$ and the diffraction data were integrated and scaled with $X D S$ (Kabsch, 2010). The other putative derivative data sets (data not shown) were measured at the ESRF, the DLS or the SLS (Swiss Light Source), Villigen, Switzerland and were processed with $X D S$.

\subsection{Phasing and crystallographic refinement}

The HKL2MAP graphical interface (Pape \& Schneider, 2004) and SHELXD (Sheldrick, 2008) were used to search all of the putative derivatives for heavy-atom sites. The only clear solution was observed for the $\mathrm{K}_{2} \mathrm{PtCl}_{4}$ derivative, yielding 23 positive hits out of 100 trials, with the best solution showing correlation coefficients $\mathrm{CC}_{\mathrm{all}}=37.6 \%$ and $\mathrm{CC}_{\text {weak }}=21.6 \%$. A total of five probable Pt sites were found with occupancies higher than 0.3 in space group $P 3_{1} 21$. However, SHELXE (Sheldrick, 2008) did not produce an interpretable electrondensity map. Therefore, the initial set of five sites was input into SHARP/autoSHARP (Bricogne et al., 2003; Vonrhein et al., 2007) for further refinement and phasing, with a phasing 
Table 1

X-ray data and structure-refinement statistics.

Values in parentheses are for the highest resolution shell.

\begin{tabular}{|c|c|c|}
\hline & Pt derivative & Native $\nmid$ \\
\hline \multicolumn{3}{|l|}{ Data collection } \\
\hline X-ray source & Beamline I04, DLS & Beamline ID14-1, ESRF $\ddagger$ \\
\hline Wavelength (§) & 0.9722 & 0.9334 \\
\hline Temperature (K) & 100 & 100 \\
\hline Detector & ADSC Quantum Q315r & ADSC Quantum Q210 \\
\hline No. of images & 250 & 1400 \\
\hline Rotation range per image $\left({ }^{\circ}\right)$ & 0.4 & 0.1 \\
\hline Space group & $P 3_{1} 21$ & $P 3_{1} 21$ \\
\hline Unit-cell parameters $(\AA)$ & $a=b=67.4, c=212.6$ & $a=b=67.17, c=210.39$ \\
\hline No. of molecules per asymmetric unit & 2 & 2 \\
\hline Matthews coefficient $V_{\mathrm{M}}\left(\AA^{3} \mathrm{Da}^{-1}\right)$ & 2.34 & 2.30 \\
\hline Solvent content $(\%)$ & 47.4 & 46.5 \\
\hline Resolution range $(\AA)$ & $50.00-2.40(2.54-2.40)$ & $33.00-1.50(1.58-1.50)$ \\
\hline Total No. of reflections & $129424(17888)$ & $744514(105892)$ \\
\hline No. of unique reflections & $41740(6489)$ & $89350(12888)$ \\
\hline Completeness $(\%)$ & $98.5(95.1)$ & $100.0(100.0)$ \\
\hline Average multiplicity & $3.1(2.8)$ & $8.3(8.2)$ \\
\hline Average $I / \sigma(I)$ & $25.76(10.91)$ & $16.8(3.1)$ \\
\hline$R_{\text {merge }}$ & $0.028(0.089)$ & $0.084(0.707)$ \\
\hline$R_{\text {p.i.m. }}$ & $0.026(0.056)$ & $0.031(0.260)$ \\
\hline Overall $B$ factor from Wilson plot $\left(\AA^{2}\right)$ & 42.6 & $15.0 \neq$ \\
\hline Anomalous correlation $\S(\%)$ & 58 & \\
\hline No. of Pt sites finally modelled & 8 & \\
\hline \multicolumn{3}{|l|}{ Refinement } \\
\hline PDB code & & 4bnd \\
\hline Resolution range $(\AA)$ & & $30.05-1.50$ \\
\hline No. of reflections & & 89249 \\
\hline No. of reflections in test set for $R_{\text {free }}$ & & 4470 \\
\hline$R_{\text {work }} / R_{\text {free }}$ & & $0.157 / 0.180$ \\
\hline \multicolumn{3}{|l|}{ No. of atoms } \\
\hline Total & & 8806 \\
\hline Protein & & 8110 \\
\hline $\mathrm{H}$ atoms (riding) & & 4075 \\
\hline Ligand & & 94 \\
\hline Water & & 602 \\
\hline \multicolumn{3}{|l|}{ Average $B$ factors $\left(\AA^{2}\right)$} \\
\hline Overall & & 19.5 \\
\hline Protein & & 18.6 \\
\hline Glycerol & & 31.4 \\
\hline Sulfate & & 14.5 \\
\hline Water & & 30.3 \\
\hline \multicolumn{3}{|l|}{ R.m.s. $\Delta B$ ( $\left(\AA^{2}\right)$} \\
\hline All bonded atoms & & 1.47 \\
\hline Bonded main-chain atoms & & 1.37 \\
\hline Bonded side-chain atoms & & 1.57 \\
\hline Atoms involved in nonbonded interactions & & 5.00 \\
\hline \multicolumn{3}{|l|}{ R.m.s. deviations } \\
\hline Bond lengths $(\AA)$ & & 0.016 \\
\hline Bond angles $\left({ }^{\circ}\right)$ & & 1.759 \\
\hline Ramachandran favoured $+\dagger(\%)$ & & 98.2 \\
\hline Ramachandran outliers $\dagger \dagger(\%)$ & & 0 \\
\hline
\end{tabular}

$\dagger$ Detailed description in Nogly et al. (2012). $\ddagger$ The information reported in Table 1 of Nogly et al. (2012) is corrected here. $\S$ Percentage of correlation between random half-sets of anomalous intensity differences computed in XDS (Kabsch, 2010). - Calculated with MOLEMAN2 (Kleywegt, 1997). †† Statistics based on RAMPAGE (Lovell et al., 2003).

power (anomalous) of 1.622 and a figure of merit (acentric) of 0.343. After density modification with SOLOMON (Abrahams \& Leslie, 1996), an interpretable electron-density map was obtained at $2.4 \AA$ resolution. Phase extension to include the higher resolution native data set was not successful, very likely owing to non-isomorphism between the Pt-derivative and native crystals. Nevertheless, a partial backbone model of the protein was built with Buccaneer (Cowtan, 2006). Based on this incomplete model, it was possible to identify the non- crystallographic symmetry (NCS) between the two molecules in the asymmetric unit. The NCS symmetry operator was used for density modification in DM (Cowtan, 1994), which further improved the quality of the calculated electron-density maps. Cycles of automated model building with Buccaneer and $A R P / w A R P$ (Langer et al., 2008) alternating with manual model building using Coot (Emsley et al., 2010) yielded a crystallographic model that was about $80 \%$ complete. Molecular replacement was then carried out with Phaser (McCoy et al., 2007) using this partial model as a search template against the higher resolution native data set. The structure was completed and refined to $1.5 \AA$ resolution using Coot and REFMAC5 (Murshudov et al., 2011). In the final refinement cycles, the model was refined using TLS groups as generated with the TLSMD server (Painter \& Merritt, 2006) and with $\mathrm{H}$ atoms added at ideal positions (Chen $e t$ al., 2010) and used as riding atoms.

The coordinates and structure factors have been deposited in the Protein Data Bank with accession code 4 bnd.

\section{Results and discussion}

\subsection{Model quality and APGM structure}

The APGM crystal soaked with $\mathrm{K}_{2} \mathrm{PtCl}_{4}$ diffracted to $2.4 \AA$ resolution and belonged to space group $P 3_{1} 21$, with unit-cell parameters $a=b=67.4$, $c=212.6 \AA$. Two protein molecules are present in the asymmetric unit, showing a root-mean-square deviation (r.m.s.d.) between $\mathrm{C}^{\alpha}$ atoms of $0.35 \AA$. The estimated solvent content is around $46.5 \%$. The structure of APGM was solved by single-wavelength anomalous dispersion (SAD) using the $\mathrm{K}_{2} \mathrm{PtCl}_{4}$ derivative. Relevant parameters for data collection and processing of the derivative data are shown in Table 1 . The final APGM model was refined to $1.5 \AA$ resolution, with an $R$ factor of $15.5 \%$ and an $R_{\text {free }}$ of $17.8 \%$ (Brünger, 1992). The crystallographic model showed good geometry and stereochemistry (PROCHECK; Laskowski et al., 1993). All amino-acid residues lie within the allowed regions of the Ramachandran plot (RAMPAGE; Lovell et al., 2003).

The electron-density maps are very well defined for the protein backbone, except for the region comprising Ile9 and 
Asp10 in molecule $B$. The side chains of 12 surface residues are not fully visible in the final $2\left|F_{\mathrm{o}}\right|-\left|F_{\mathrm{c}}\right|$ electron-density map contoured at $1 \sigma$, likely owing to their mobility, and 26 side chains were modelled with alternate conformations, including the catalytic residue Asp8. A blob of residual electron density was observed near the active site of the enzyme (cap domain). Based on its tetrahedral shape and the nearby residues, a sulfate ion was fitted into the blob and further refined. Six glycerol molecules were added to the model, one of which is close to the sulfate ion in chain $B$. The final APGM model comprises 404 amino-acid residues (252 per chain), 602 water molecules, two sulfate ions and six glycerol molecules.

Each molecule comprises a core domain (residues 1-88 and 189-252), typical of the HADSF, and a small cap domain (residues 93-183) as illustrated in Fig. 1(a). The core domain consists of a seven-stranded $\beta$-sheet $(\beta 5, \beta 4, \beta 3$, $\beta 2, \beta 1, \beta 10$ and $\beta 11$ ) flanked by five $\alpha$ helices $(\alpha 6, \alpha 7, \alpha 8, \alpha 1$ and $\alpha 2)$. The cap domain is constituted by a four-stranded antiparallel $\beta$-sheet $(\beta 6, \beta 7, \beta 9$ and $\beta 8)$ with three $\alpha$-helices located on one face $(\alpha 3, \alpha 5$ and $\alpha 4)$. The cap and core domains are connected by two linker

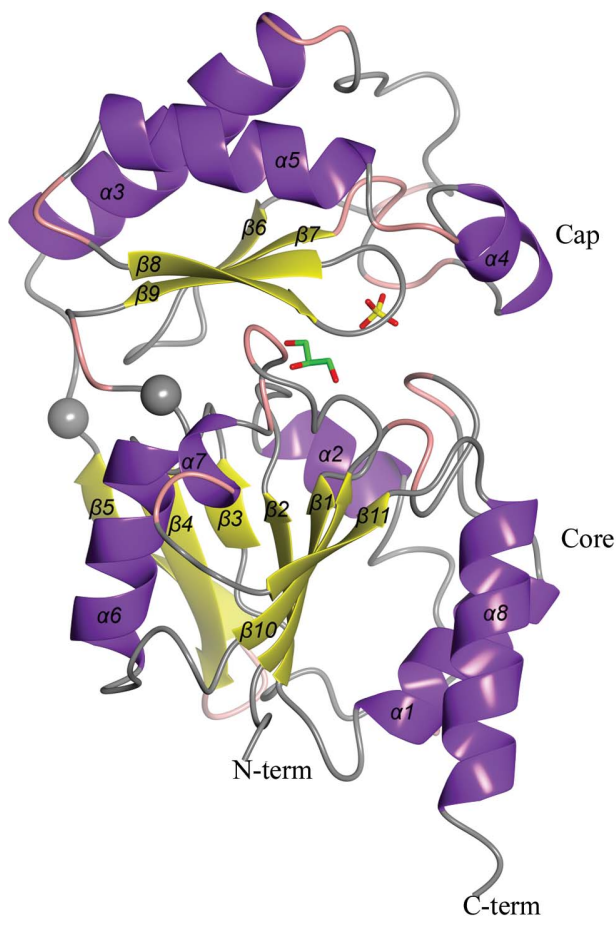

(a)

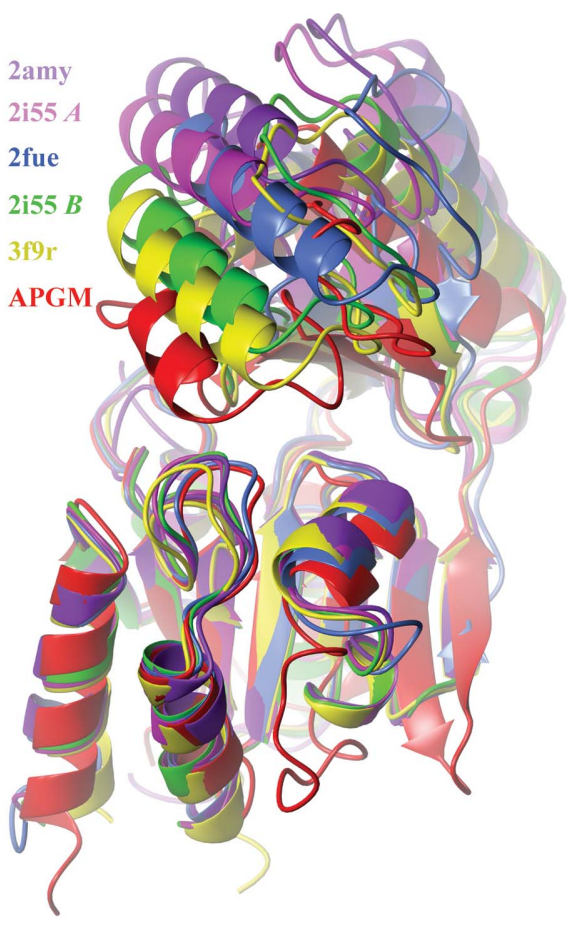

(b)

Figure 1

Structure of APGM and comparison with PMMs. (a) APGM ribbon representation (molecule $B$ ) coloured according to the secondary-structure elements assigned by DSSP (Kabsch \& Sander, 1983) and HingeProt ( $\beta$-strands, yellow; $\alpha$-helices, magenta; turns, pink; hinges, spheres). Glycerol and sulfate located at the interface of the core and cap domains are drawn in stick mode. Atom colour codes: C, green; O, red; S, yellow. Figs. 1 and 3 were drawn with CCP4mg (McNicholas et al., 2011). (b) Structural superposition of APGM and PMMs, aligned by the core domains (with SwissPDBViewer; Guex \& Peitsch, 1997), showing the different conformations of the cap domain relative to the core domain, with APGM being most closed (depicted in red; chain $A$ ). The PDB code identifies the PMM structures: 2i55, L. mexicana PMM; 2fue, human PMM1, 2amy; human PMM2; 3f9r, T. brucei PMM. loops (89-92 and 184-188) that may act as a hinge joint. Indeed, HingeProt (Emekli et al., 2008) predicts a more mobile region around residues 88 and 188 (Fig. 1a).

The APGM active site is located at the interface between the core and cap domains. This interface comprises several positively charged residues, such as Lys17 and Lys44 in the core domain and Arg128, Lys140 and Lys146 in the cap domain, which are proposed to keep the enzyme in an open conformation, a feature that is also observed in PMMs (Silvaggi et al., 2006). In the structure of human PMM1 the substrate is bound to the cap domain in the cap-open conformation, which was suggested to represent the first encounter complex (Silvaggi et al., 2006). Upon binding of the negatively charged substrate (i.e. $\alpha$-glucose-1-phosphate) in the cap domain, the electrostatic repulsion between the cap and core domains may be shielded, favouring the closure of the cap domain and the proper positioning of the substrate with respect to catalytic residues in the core domain. A closed cap domain also provides a solvent-excluded environment. The core domain contains the conserved catalytic residues of the HADSF (see below for further details), while the cap domain has a more variable size and structure and thus is proposed to be involved in substrate recognition. The topology and location of the cap domain allows the classifi- cation of APGM into the type IIb subfamily of the HADSF (Allen \& Dunaway-Mariano, 2004).

\subsection{Similar structures}

The overall structure of APGM is similar to eukaryotic phosphomannomutases (PMMs), despite their low sequence identity (up to $24 \%$ ). The four best hits from $D A L I$ were (the PDB entry, $Z$-score and r.m.s.d. for $\mathrm{C}^{\alpha}$ superposition are indicated for each hit): Leishmania mexicana PMM (2i55, 23.7, 2.9 Å; Kedzierski et al., 2006), Trypanosoma brucei PMM (3f9r, 24.2, 3.0 Å; Structural Genomics Consortium, unpublished work), human PMM1 (2fue, 20.1, 4.1 А; Silvaggi et al., 2006) and human PMM2 (2amy, 17.4, $5.3 \AA$ A; Center for Eukaryotic Structural Genomics, unpublished work). Superposition of APGM with these four crystal structures shows that the cap domain is more tightly closed in APGM than in PMMs (Fig. 1b). In contrast, the structure of human PMM2 (PDB entry 2amy) without any ligand bound at the domain interface has the cap domain furthest away from the core domain. The structure of leishmanial PMM (PDB entry 2i55) may well illustrate the cap closure upon ligand binding as a result of alleviated electrostatic repulsion between the domains: polypeptide chain $A$ has no ligand at the active site, 
whereas chain $B$ has a ligand bound and thus is in a more closed conformation. The ligand, $\beta$-glucose 1,6-bisphosphate, extensively interacts with both domains. Remarkably, the structure of human PMM1 bound to mannose 1-phosphate (PDB entry 2fue) was captured in a cap-open state with the substrate interacting mainly with residues from the cap domain. This structure was obtained by soaking the ligand into pre-formed crystals, which was proposed to restrict the movements of the cap domain within the crystal lattice (Silvaggi et al., 2006).

Binding of sulfate at the phosphoryl binding site (as in APGM and PDB entry 3f9r), rather than larger ligands (as in PDB entry $2 \mathrm{i} 55$ and 2 fue), is likely to result in a more closed conformation of the cap domain. However, the structure of T. brucei PMM with sulfate displays a more open conformation than APGM. The positive patch at the interface of T. brucei PMM comprises Arg19 and Lys50 in the core domain, and Arg122, Arg133 and Arg140 in the cap domain and is highly conserved among PMMs. However, in APGM the Lys140 side chain is oriented towards the solvent, which could mitigate the electrostatic repulsion between the positively charged residues at the APGM domain interface, thus allowing a more tightly closed cap conformation.

APGM shows several insertion regions with regard to PMMs (Fig. 2), which are mainly located at the molecular surface of the core domain, namely between helix $\alpha 2$ and strand $\beta 3$, between strands $\beta 4$ and $\beta 5$, between helix $\alpha 3$ and strand $\beta 6$ (cap domain), and between helix $\alpha 6$ and strand $\beta 10$. These insertions are not expected to cause major structural differences in APGM compared with PMMs, except for that at the cap domain, as discussed below.

\subsection{Quaternary structure}

The molecular mass of APGM estimated by size-exclusion chromatography was $55 \mathrm{kDa}$, indicating the presence of a dimeric form in solution. The dimeric assembly proposed by PISA (Krissinel \& Henrick, 2007) corresponds to a crystal contact in the APGM structure (Fig. 3a). The complexation significance score (CSS) for the polypeptide suggests that
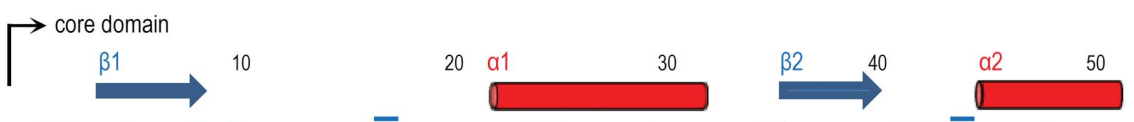

60

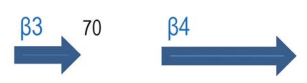

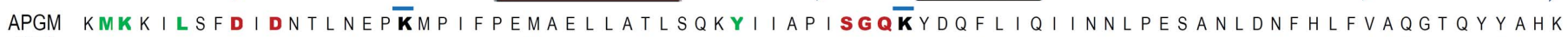

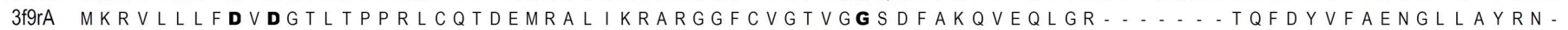

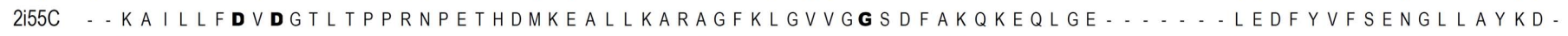

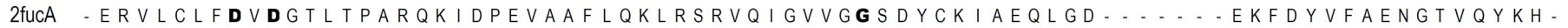

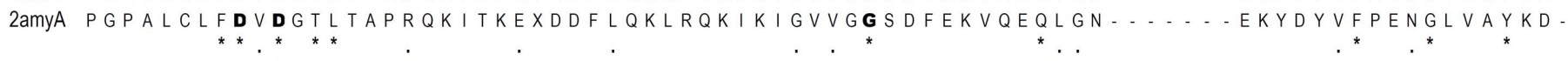

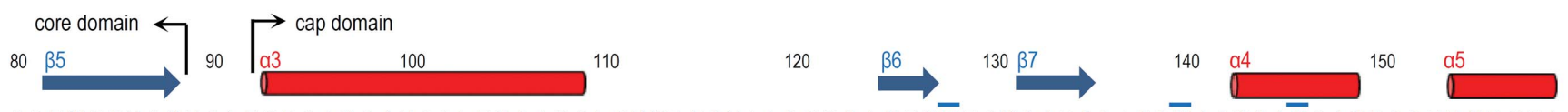

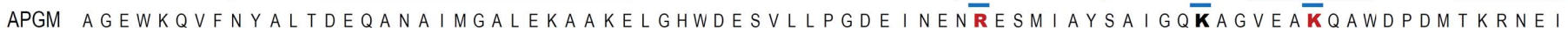
3f9rA - - G LE I HRQSLLNDR I VKFVKKTLRLIADL - D I PVQR - G T FVEYRNGMINVSPIGRNCSQAERDEFEVYDRVRASL

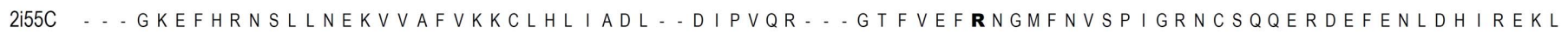

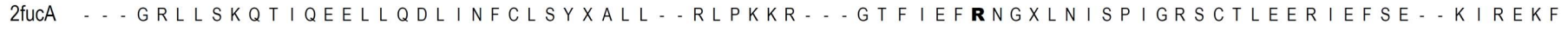

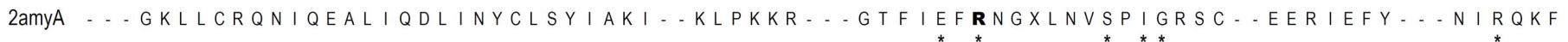
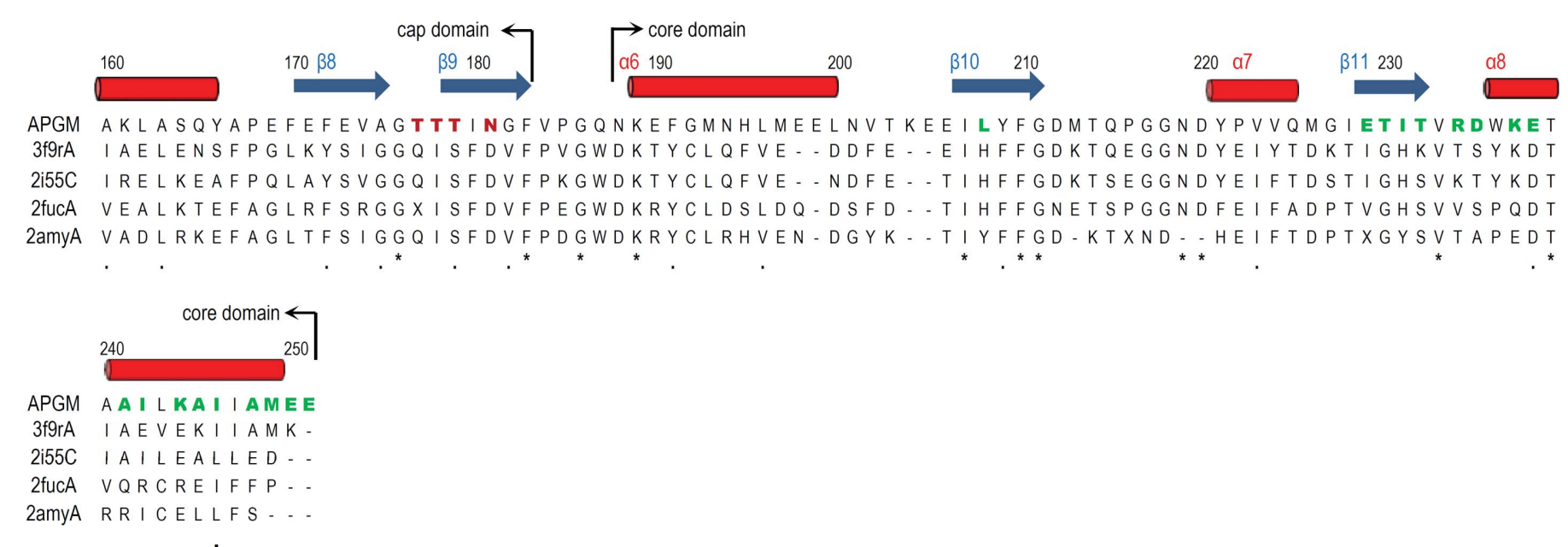

Figure 2

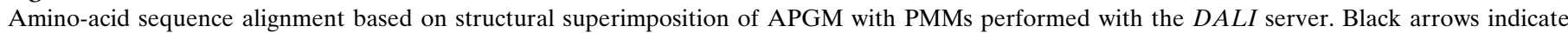

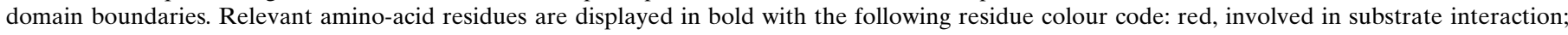

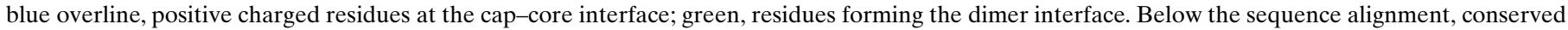
residues are indicated by an asterisk (*) and similar residues by a dot (.). Blue arrows represent $\beta$-strands and red tubes represent $\alpha$-helices. 
the interface plays an essential role in complex formation. The occluded area at this interface is approximately $1000 \AA^{2}$ (around 5\% of the total area) and is formed by hydrophobic amino-acid residues mainly located in the C-terminal helix $\alpha 8$ and hydrophilic residues sited on the stretch between $\beta 11$ and $\alpha 8$ (core domain). It also includes waters mediating hydrogen bonds between the two molecules. This dimeric arrangement should represent the biological unit of APGM and may be relevant to the enzyme function, as it brings the active sites within the dimer into proximity. It is worth noting that in APGM helix $\alpha 8$ is shifted further away from the $\beta$-sheet of the same monomer towards $\alpha 8$ and $\beta 11$ of the other monomer than in PMMs and thus forms a hydrophobic groove accommodating residues sited on $\alpha 8$ of the other molecule within the dimer.

The dimeric assembly proposed for $L$. mexicana PMM shows a different interface formed exclusively by amino-acid residues from the cap domains (Kedzierski et al., 2006). The dimer formation includes a helix-helix interaction between the first helices $(\alpha 3)$ and an antiparallel $\beta$-sheet interaction between the first strands $(\beta 6)$ of the two cap domains, which extends the solvent-exposed face of the $\beta$-sheet, placing the two active sites adjacent to each other. This interface is also conserved in human PMMs (Silvaggi et al., 2006) and likely in T. brucei PMM (as predicted by the PISA server). Structurebased sequence alignment of APGM with these four homologous PMMs shows a five-residue-insertion in this region of the cap domain between helix $\alpha 3$ and strand $\beta 6$ (Fig. 2). Structural superposition reveals that the loop comprising Leu110-Glu123 adopts a different conformation in APGM, including a protrusion (Glu115-Glu123) facing outwards, which may explain why APGM cannot form the same dimeric assembly as the aforementioned PMM structures (Fig. 3b).

\subsection{Active site and catalytic mechanism}

APGM catalyzes the interconversion of $\alpha$-glucose 1-phosphate and glucose 6-phosphate via a phophorylated enzyme intermediate, as proposed for other phosphoryl-transfer enzymes of the HADSF (Dai et al., 2006; Kedzierski et al., 2006). The enzyme first has to be activated by the transfer of a phosphate from the $\alpha$-glucose 1,6-bisphosphate cofactor to the enzyme aspartyl nucleophile (Asp8 in APGM), producing an aspartyl phosphate (Supplementary Fig. S1 ${ }^{\mathbf{1}}$ ). The substrate ( $\alpha$-glucose 1-phosphate) then binds, forming a covalent bond between the substrate $\mathrm{C} 6$ hydroxyl $\mathrm{O}$ atom and the aspartylphosphate $\mathrm{P}$ atom. An $\alpha$-glucose-1,6-bisphosphate intermediate is generated, which then needs to be released from the enzyme and rebound in a reoriented position so that the 1-phosphate is transferred to the aspartyl nucleophile, replenishing the activated form of the enzyme and forming the final product (glucose 6-phosphate). Release of the phosphate from the bisphospho-carbohydrate intermediate to the aspartyl group is facilitated by another acid/base group (Asp10). The catalytic

\footnotetext{
${ }^{1}$ Supplementary material has been deposited in the IUCr electronic archive (Reference: DZ5291). Services for accessing this material are described at the back of the journal.
}

nucleophile (Asp8) usually coordinates a magnesium ion along with other residues and water molecules. No metal ion is observed in the APGM crystal structure and no $\mathrm{Mg}^{2+}$ ions were present in the crystallization buffer.

Although the relevance of the APGM dimer to its catalytic activity has not yet been demonstrated, in this arrangement the two active sites are placed face to face with the sulfate ions $\sim 38 \AA$ apart. Interestingly, an extended positively charged patch is observed in a groove between the active sites, comprising two arginine residues $(\operatorname{Arg} 234 A / B)$ and two lysine residues $($ Lys237 $A / B)$. The positive charge between the active sites may prolong the presence of the intermediate within the dimer instead of its release into the solution. A functional role has also been proposed for the dimeric forms of human and leishmanial phosphomannomutases (Kedzierski et al., 2006; Silvaggi et al., 2006).

The unsuccessful attempts to obtain the structure of APGM in complex with substrate analogues either by crystal soaking or cocrystallization may be due to the presence of sulfate in the APGM active site (Lu et al., 2005). Sulfate exchange for malonate was also attempted, but resulted in crystal degra-

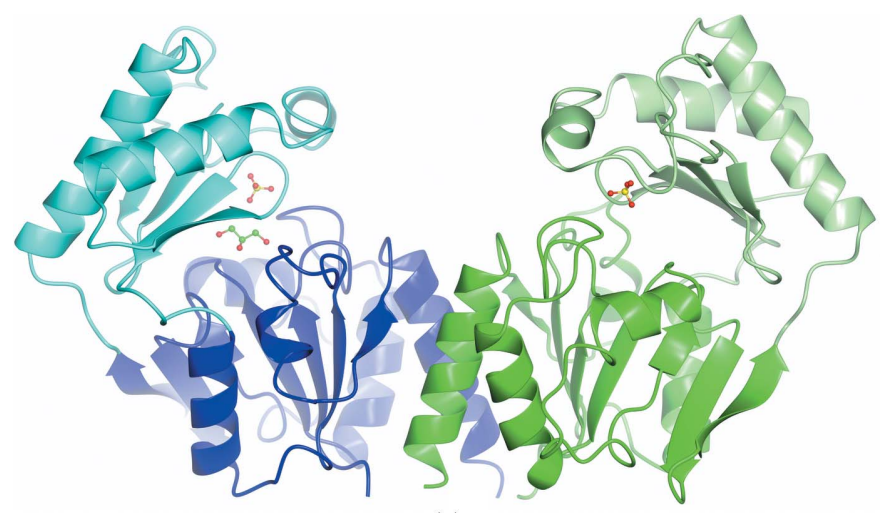

(a)

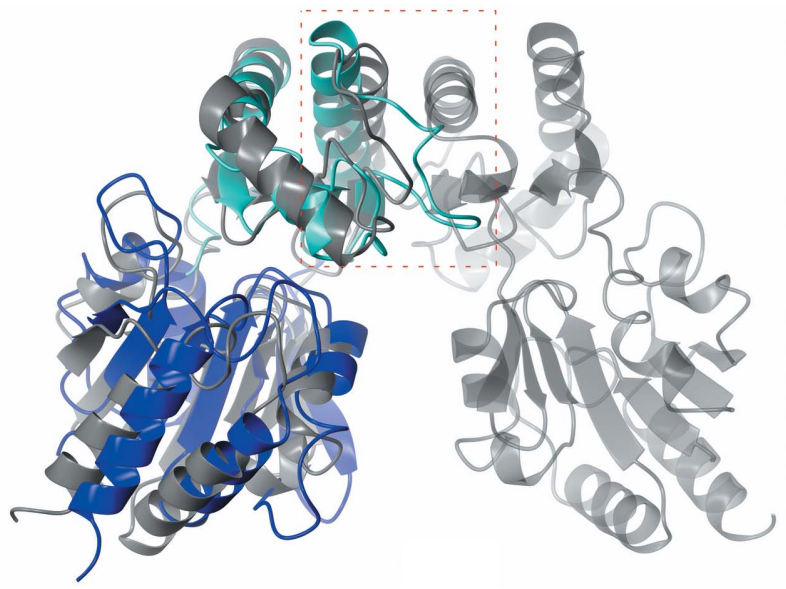

(b)

Figure 3

Comparison of APGM and PMM dimers. (a) The APGM dimer identified by PISA. Molecule $B$ is coloured blue and molecule $A$ is coloured green (dark colour for the core domain and light colour for the cap domain). Sulfate and glycerol are located at the substrate-binding site. (b) Dimer of leishmanial PMM (grey) illustrating the conserved dimeric interface of eukaryotic PMMs. Superimposition with one molecule of APGM (core, dark blue; cap, cyan) by the cap domain, highlighting the longer loop in APGM. 


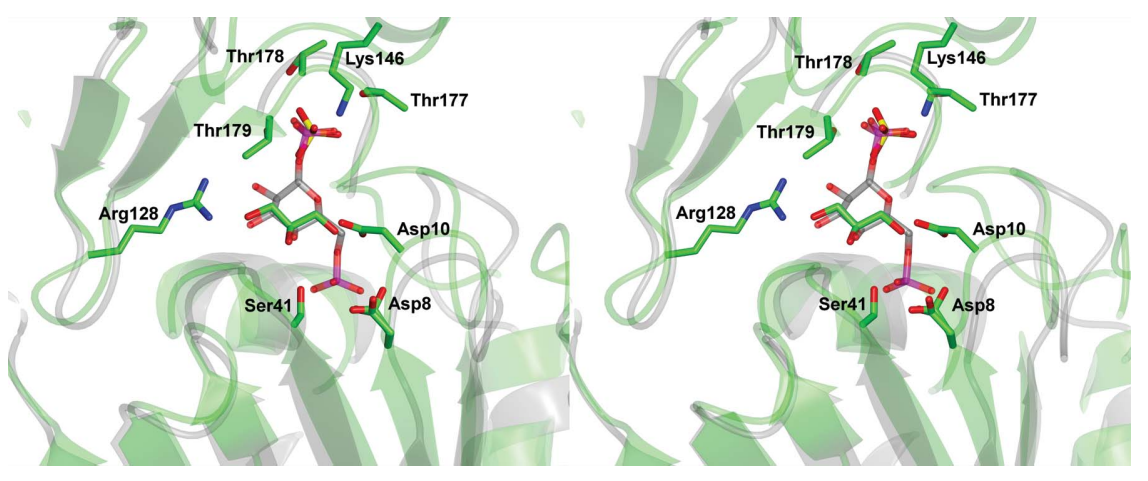

Figure 4

Stereoview of the APGM active site. Superposition of APGM and leishmanial PMM structures, highlighting the alignment of glycerol and sulfate (APGM; C, green; alternate conformation of Asp8) with $\beta$-glucose 1,6-bisphosphate (leishmanial PMM; C, grey; P, magenta). (cap domain) is mainly composed by two arginines and one serine (Arg143, Arg150 and Ser188; human PMM1 numbering).

In addition, the glycerol found near the sulfate ion (molecule $B$, closest distance of $4.1 \AA$ ) aligns very well with part of the pyranose ring of $\beta$-glucose 1,6-biphosphate bound to the leishmanial PMM structure. In APGM, the two hydroxyl groups of glycerol that mimic part of the sugar moiety establish hydrogen bonds to the side chains of Arg128 and Asn181 (corresponding to Arg122 and Asp180 in leishmanial PMM), the mainchain amide-group peptide hydrogen of Gly42 and a few water molecules. It is worth noting that in the APGM structure the side chain of Asp10 is hydrogen bonded to one

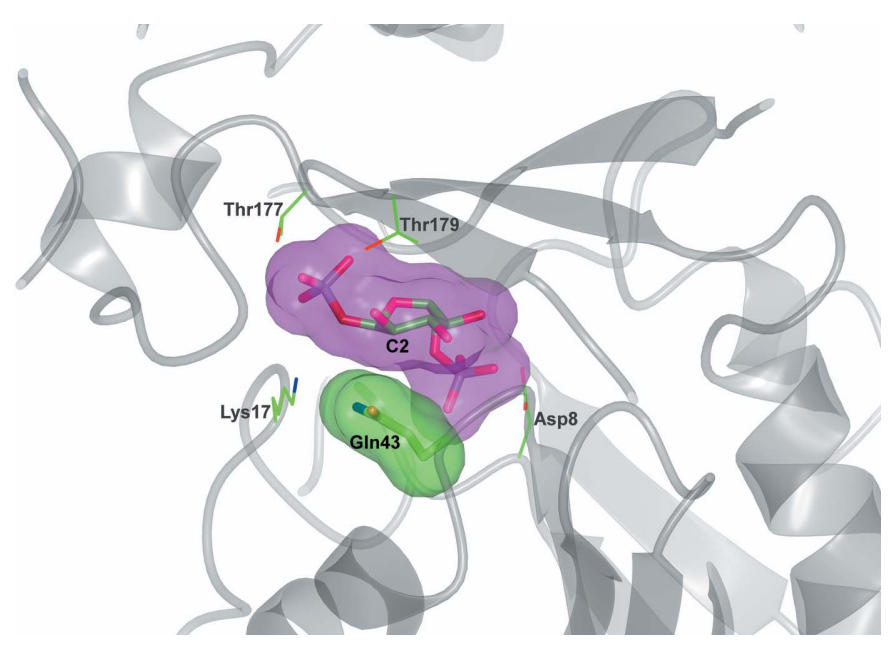

Figure 5

Active site of APGM fitted with $\alpha$-glucose 1,6-bisphosphate (manual fitting followed by structure idealization). van der Waals surfaces of the Gln43 side chain and phosphosugar ligand are coloured green and pink, respectively, highlighting the steric restrictions imposed by Gln 43 below the $\mathrm{C} 2$ atom of the sugar ring.

dation (McPherson, 2001). Nevertheless, important information on substrate binding can be derived from the homologous PMM structures, in particular those of human PMM1 in complex with $\alpha$-mannose 1-phosphate and leishmanial PMM in complex with $\beta$-glucose 1,6-bisphosphate. In fact, the sulfate bound at the active site of APGM molecules $A$ and $B$ overlays quite well with the phosphoryl group (bound to $\mathrm{C} 1$ ) of $\beta$-glucose 1,6-bisphosphate bound to the L. mexicana PMM structure (Fig. 4). Moreover, if the cap domains of the APGM and human PMM1 structures are superposed, the sulfate ion in APGM also matches the position of the phosphoryl group of $\alpha$-mannose 1-phosphate. In the APGM structure, the sulfate is hydrogen bonded to the main-chain amide and sidechain hydroxyl groups of Thr177, Thr178 and Thr179, Lys146 and a few water molecules. It is also close to Gln43 from the core domain $(\sim 4.1$ and $3.1 \AA$ in molecules $A$ and $B$, respectively). In all four eukaryotic PMM structures from the $D A L I$ survey, this distal sulfate/substrate phosphoryl-binding pocket glycerol hydroxyl (corresponding to the C6 hydroxyl of the PMM ligand) in molecule $B$, while in molecule $A$, in the absence of glycerol, it is shifted $2.8 \AA$ towards the interior of the active-site pocket. In contrast, in PMMs the corresponding Asp side chain is further away from the ligand $(\sim 7 \AA$ from the C6 atom of glucose in leishmanial PMM), with the exception of human PMM1, in which the acid/base Asp is in a conformation corresponding to molecule $B$ of APGM. The mobility of Asp10, which plays an important role in the catalytic mechanism, is clearly evident from the different positions adopted by this acid/base residue in APGM and PMMs. Also, the poorly defined electron density around the backbones of Ile9 and Asp10 in molecule $B$ suggests flexibility and possibly alternate conformations. In addition, in the active site of molecule $B$ the side chain of Asp8 adopts alternate conformations; two water molecules are observed to mediate contacts between this aspartate and glycerol.

Moreover, we have fitted $\alpha$-glucose 1,6-bisphosphate into APGM, based on the previous superposition with leishmanial PMM, and a model with reasonable stereochemistry was obtained except for Ser41, which lies too close $(1.3 \AA)$ to the phosphoryl group (Fig. 4). This residue is replaced by a conserved glycine in PMMs. A shift of the molecule towards Asp8 and a slight rotation around the phosphodiester bond followed by geometry idealization with REFMAC5 (Murshudov et al., 2011) provided a good fit of the ligand into the binding pocket (Fig. 5).

A relevant point to discuss is the strict specificity of APGM towards $\alpha$-glucose 1-phosphate, in contrast to PMMs, which can use both $\alpha$-glucose 1-phosphate and mannose 1-phosphate as substrates. The only difference between these two sugars is the position of the hydroxyl group at $\mathrm{C} 2$ of the pyranose ring (equatorial in $\alpha$-glucose 1-phosphate and axial in mannose 1-phosphate). Structural superimposition of APGM with leishmanial PMM shows that the side chain of Gln43 in APGM lies in close proximity to $\mathrm{C} 2$ of the pyranose ring of $\alpha$-glucose 1,6 -bisphosphate $\left(\mathrm{G} \ln 43 \mathrm{~N}^{\varepsilon 2}\right.$ is only $2.3 \AA$ away from $\mathrm{C} 2$ ). This residue restricts the space under the sugar ring, which may be needed to accommodate the phosphomannose (Fig. 5). If the hydroxyl group of $\mathrm{C} 2$ is in the axial position, 
as in mannose 1,6-bisphosphate, it would clash with the side chain of Gln43. This steric hindrance in the presence of phosphomannose may prevent the full closure of the cap over the core domain and restrict the proper positioning of the phosphomannose to react with the catalytic residues in the core domain. This Gln43 is replaced by a strictly conserved serine in PMMs and no steric hindrance is observed. This amino-acid substitution is likely to be responsible for the strict specificity of APGM towards $\alpha$-glucose 1-phosphate.

In addition, the crystal structure of human PMM1 with mannose 1-phosphate was proposed to represent the first encounter state of the ligand with the enzyme (Silvaggi et al., 2006). The cap domain is found in an open conformation and the residues engaged in the interactions with the ligand are mainly from the cap domain. In this structure, the hydroxyl group at $\mathrm{C} 2$ of the pyranose ring is hydrogen bonded to Arg132, Asn137 and Asp190. The corresponding residues in APGM are Arg128, Ala133 and Asn181. The substitution of Asn by Ala affects the enzyme-substrate interactions, as no hydrogen bond can be established by Ala in APGM. Because the cap domain accounts for substrate recognition and binding, the weaker interactions with mannose 1-phosphate in APGM may also contribute to its specificity towards $\alpha$-glucose 1-phosphate.

\section{Conclusions}

Here, we have presented the first high-resolution structure of $L$. lactis $\alpha$-phosphoglucomutase (APGM) with substratemimicking sulfate and glycerol bound at the active site. Unlike other bacterial $\alpha$-phosphoglucomutases, APGM belongs to the haloacid dehalogenase superfamily with structural features showing the characteristic catalytic core and mobile cap domains. The enzyme cap domain is found in a tightly closed conformation. APGM is found to be a dimer in solution as well as in the crystal structure, which might be relevant to the enzyme efficiency; however, the dimer interface differs from that found in eukaryotic PMMs. Despite low sequence identity, APGM shares structural homology with eukaryotic phosphomannomutases, although it can only utilize $\alpha$-glucose 1-phosphate and not its stereoisomer $\alpha$-mannose 1-phosphate, unlike PMMs. Structural superposition of APGM and PMMs highlights their similarity and differences. A relevant difference seems to be the replacement of the conserved Gly and Ser residues in PMMs by Ser41 and Gln43, respectively, in APGM, which adds steric constraints at the APGM active site. The presented work provides the first structural insights into this atypical $\alpha$-phosphoglucomutase.

We are grateful to Carlos Frazao, Colin McVey, Cláudio Soares, Alekos Athanasiadis and Pikyee Ma for careful reading of this manuscript, helpful discussions and collection of diffraction data. This work was supported by Fundação para a Ciência e a Tecnologia (FCT) through grants PTDC/BIAMIC/099963/2008, PTDC/BIA-PRO/103718/2008 and PEstOE/EQB/LA0004/2011 and by an EU Grant (FP7/2007-2013, No. 211800) to MA and PN. We acknowledge the Diamond
Light Source, ESRF and SLS synchrotrons for financial support for data collection.

\section{References}

Abrahams, J. P. \& Leslie, A. G. W. (1996). Acta Cryst. D52, 30-42.

Allen, K. N. \& Dunaway-Mariano, D. (2004). Trends Biochem. Sci. 29, 495-503.

Allen, K. N. \& Dunaway-Mariano, D. (2009). Curr. Opin. Struct. Biol. 19, 658-665.

Andersson, U., Levander, F. \& Rådström, P. (2001). J. Biol. Chem. 276, 42707-42713.

Battye, T. G. G., Kontogiannis, L., Johnson, O., Powell, H. R. \& Leslie, A. G. W. (2011). Acta Cryst. D67, 271-281.

Bricogne, G., Vonrhein, C., Flensburg, C., Schiltz, M. \& Paciorek, W. (2003). Acta Cryst. D59, 2023-2030.

Brünger, A. T. (1992). Nature (London), 355, 472-475.

Chen, V. B., Arendall, W. B., Headd, J. J., Keedy, D. A., Immormino, R. M., Kapral, G. J., Murray, L. W., Richardson, J. S. \& Richardson, D. C. (2010). Acta Cryst. D66, 12-21.

Cowtan, K. (1994). Jnt CCP4/ESF-EACBM Newsl. Protein Crystallogr. 31, 34-38.

Cowtan, K. (2006). Acta Cryst. D62, 1002-1011.

Dai, J., Wang, L., Allen, K. N., Radstrom, P. \& Dunaway-Mariano, D. (2006). Biochemistry, 45, 7818-7824.

Delcour, J., Ferain, T., Deghorain, M., Palumbo, E. \& Hols, P. (1999). Antonie Van Leeuwenhoek, 76, 159-184.

Emekli, U., Schneidman-Duhovny, D., Wolfson, H. J., Nussinov, R. \& Haliloglu, T. (2008). Proteins, 70, 1219-1227.

Emsley, P., Lohkamp, B., Scott, W. G. \& Cowtan, K. (2010). Acta Cryst. D66, 486-501.

Evans, P. R. (2011). Acta Cryst. D67, 282-292.

Fettke, J., Hejazi, M., Smirnova, J., Hochel, E., Stage, M. \& Steup, M. (2009). J. Exp. Bot. 60, 2907-2922.

Grossiord, B., Vaughan, E. E., Luesink, E. \& de Vos, W. M. (1998). Lait, 78, 77-84.

Guex, N. \& Peitsch, M. C. (1997). Electrophoresis, 18, 2714-2723.

Kabsch, W. (2010). Acta Cryst. D66, 125-132.

Kabsch, W. \& Sander, C. (1983). Biopolymers, 22, 2577-2637.

Kedzierski, L., Malby, R. L., Smith, B. J., Perugini, M. A., Hodder, A. N., Ilg, T., Colman, P. M. \& Handman, E. (2006). J. Mol. Biol. 363, 215-227.

Kleerebezem, M., van Kranenburg, R., Tuinier, R., Boels, I. C., Zoon, P., Looijesteijn, E., Hugenholtz, J. \& de Vos, W. M. (1999). Antonie Van Leeuwenhoek, 76, 357-365.

Kleywegt, G. J. (1997). J. Mol. Biol. 273, 371-376.

Krissinel, E. \& Henrick, K. (2007). J. Mol. Biol. 372, 774-797.

Lahiri, S. D., Zhang, G., Dai, J., Dunaway-Mariano, D. \& Allen, K. N. (2004). Biochemistry, 43, 2812-2820.

Lahiri, S. D., Zhang, G., Dunaway-Mariano, D. \& Allen, K. N. (2002). Biochemistry, 41, 8351-8359.

Langer, G., Cohen, S. X., Lamzin, V. S. \& Perrakis, A. (2008). Nature Protoc. 3, 1171-1179.

Laskowski, R. A., MacArthur, M. W., Moss, D. S. \& Thornton, J. M. (1993). J. Appl. Cryst. 26, 283-291.

Levander, F., Andersson, U. \& Radstrom, P. (2001). Appl. Environ. Microbiol. 67, 4546-4553.

Lovell, S. C., Davis, I. W., Arendall, W. B., de Bakker, P. I. W., Word, J. M., Prisant, M. G., Richardson, J. S. \& Richardson, D. C. (2003). Proteins, 50, 437-450.

Lu, Z., Dunaway-Mariano, D. \& Allen, K. N. (2005). Biochemistry, 44, 8684-8696.

McCoy, A. J., Grosse-Kunstleve, R. W., Adams, P. D., Winn, M. D., Storoni, L. C. \& Read, R. J. (2007). J. Appl. Cryst. 40, 658-674.

McNicholas, S., Potterton, E., Wilson, K. S. \& Noble, M. E. M. (2011). Acta Cryst. D67, 386-394.

McPherson, A. (2001). Protein Sci. 10, 418-422. 
Murshudov, G. N., Skubák, P., Lebedev, A. A., Pannu, N. S., Steiner, R. A., Nicholls, R. A., Winn, M. D., Long, F. \& Vagin, A. A. (2011). Acta Cryst. D67, 355-367.

Neves, A. R., Pool, W. A., Castro, R., Mingote, A., Santos, F., Kok, J., Kuipers, O. P. \& Santos, H. (2006). J. Biol. Chem. 281, 36864-36873.

Nogly, P., Castro, R., de Rosa, M., Neves, A. R., Santos, H. \& Archer, M. (2012). Acta Cryst. F68, 1113-1115.

Painter, J. \& Merritt, E. A. (2006). Acta Cryst. D62, 439-450.

Pape, T. \& Schneider, T. R. (2004). J. Appl. Cryst. 37, 843-844.

Paterson, G. K., Cone, D. B., Peters, S. E. \& Maskell, D. J. (2009). Microbiology, 155, 3403-3410.

Pirard, M., Achouri, Y., Collet, J.-F., Schollen, E., Matthijs, G. \& Schaftingen, E. V. (1999). Biochem. J. 339, 201-207.
Plant, L., Sundqvist, J., Zughaier, S., Lovkvist, L., Stephens, D. S. \& Jonsson, A. (2006). Infect. Immun. 74, 1360-1367.

Shackelford, G. S., Regni, C. A. \& Beamer, L. J. (2004). Protein Sci. 13, 2130-2138.

Sheldrick, G. M. (2008). Acta Cryst. A64, 112-122.

Silvaggi, N. R., Zhang, C., Lu, Z., Dai, J., Dunaway-Mariano, D. \& Allen, K. N. (2006). J. Biol. Chem. 281, 14918-14926.

Villar-Palasi, C. \& Larner, J. (1970). Annu. Rev. Biochem. 39, 639-672.

Vonrhein, C., Blanc, E., Roversi, P. \& Bricogne, G. (2007). Methods Mol. Biol. 364, 215-230.

Whitehouse, D. B., Tomkins, J., Lovegrove, J. U., Hopkinson, D. A. \& McMillan, W. O. (1998). Mol. Biol. Evol. 15, 456-462. 